\title{
The Correlation Between Clinical Characteristics and Radionuclide Salivagram Findings in Patients With Brain Lesions: A Preliminary Study
}

\author{
Donghwi Park, MD, Seung Beom Woo, MD, Dae Hee Lee, MD, Kwang Jae Yu, MD, \\ Ju Young Cho, MD, Jong Min Kim, MD, Zeeihn Lee, MD, PhD
}

Department of Rehabilitation Medicine, Daegu Fatima Hospital, Daegu, Korea

\begin{abstract}
Objective To evaluate the correlation between radionuclide salivagram findings and clinical characteristics in dysphagic patients with brain lesions.

Methods The medical records of 35 dysphagic patients with brain lesions who simultaneously underwent both a videofluoroscopic swallowing study (VFSS) and radionuclide salivagram were analyzed retrospectively. The subjects were divided into two groups according to the presence of aspiration on a salivagram (group A, patients with aspiration on the salivagram; group B, patients with no aspiration on the salivagram). The differences between clinical characteristics and VFSS findings (penetration-aspiration scale [PAS]) between the two groups were analyzed.

Results Eleven out of 35 patients displayed salivary aspiration on the radionuclide salivagram. There were no significant differences between the two groups according to age, sex, disease duration, PAS on VFSS and feeding methods ( $\mathrm{p} \geq 0.05)$. The incidence of aspiration pneumonia was significantly higher in group A. In a multivariate logistic regression analysis with forward stepwise method, the Mini-Mental State Examination (MMSE) score was the only significant parameter in predicting positive findings in salivagrams (odds ratio $=0.760$; $95 \%$ confidence interval [CI], 0.625-0.923; $\mathrm{p}=0.006$ ). The area under the receiver operating characteristic curve (AUC) of the MMSE score for positive detection in salivagrams was 0.855 (95\% CI, 0.689-0.953; $\mathrm{p}<0.0001$ ). The optimal cut-off value was 7 for the MMSE score (sensitivity $72.73 \%$, specificity $100 \%$ ).

Conclusion In patients with brain lesions who complain of dysphagia, the MMSE score was correlated with salivary aspiration. If patients present with a score of 7 or less on the MMSE, performing a radionuclide salivagram may helpful for early detection of patients at high risk of aspiration pneumonia induced from salivary aspiration.
\end{abstract}

Keywords Sialorrhea, Deglutition, VFSS, Aspiration pneumonia, Cognition

Received April 14, 2017; Accepted June 2, 2017

Corresponding author: Seung Beom Woo

Department of Rehabilitation Medicine, Daegu Fatima Hospital, 99 Ayang-ro, Dong-gu, Daegu 41199, Korea. Tel: +82-53-940-7821, Fax: +82-53-9547417, E-mail:wsb0301@gmail.com

ORCID: Donghwi Park (http://orcid.org/0000-0002-7724-4682); Seung Beom Woo (http://orcid.org/0000-0002-0580-8696); Dae Hee Lee (http://orcid. org/0000-0003-2069-2318); Kwang Jae Yu (http://orcid.org/0000-0003-4929-6677); Ju Young Cho (http://orcid.org/0000-0002-7843-5480); Jong Min Kim (http://orcid.org/0000-0002-4748-2030); Zeeihn Lee (http://orcid.org/0000-0002-4748-2030).

(c) This is an open-access article distributed under the terms of the Creative Commons Attribution Non-Commercial License (http://creativecommons.org/ licenses/by-nc/4.0) which permits unrestricted noncommercial use, distribution, and reproduction in any medium, provided the original work is properly cited. Copyright ( 2017 by Korean Academy of Rehabilitation Medicine 


\section{INTRODUCTION}

Aspiration following a brain injury has been associated with pneumonia, sepsis and death. Among complications, pneumonia is the major cause of death during the acute and chronic phases of brain injury $[1,2]$. Aspiration is the misdirection of oropharyngeal or gastric contents into the larynx and lower respiratory tract [3]. The causes of aspiration include swallowing dysfunction, gastroesophageal reflux, and salivary aspiration. There are several methods for assessing aspiration, such as the videofluoroscopic swallowing study (VFSS), fiberoptic endoscopic evaluation of swallowing, and radionuclide salivagram.

Among them, VFSS has become the most commonly utilized method for evaluating swallowing function and aspiration [4] because of its ability to evaluate the oropharyngeal and esophageal phases of swallowing. However, the previous studies that used VFSS reported significant false-negative results in predicting aspiration pneumonia $[5,6]$.

The radionuclide salivagram detects salivary aspiration $[7,8]$. In a radionuclide salivagram, a small amount of radiolabeled colloid is typically placed in the patient's mouth, allowing it to mix with oral secretions. Then, serial images are recorded to ascertain the existence of radioactivity in the tracheobronchial tree, which would indicate aspiration. This test may be useful for the early detection of salivary aspiration that can induce aspira- tion pneumonia. Despite this function, the use of radionuclide salivagram as a routine diagnostic test for patients with brain lesions with complaints of dysphagia has not gained wide acceptance because there has not yet been accurate outcomes. In this study, therefore, a radionuclide salivagram in patients with brain lesions was investigated by examining the correlation between the findings of a radionuclide salivagram and patients' clinical characteristics.

\section{MATERIALS AND METHODS}

\section{Subjects}

A retrospective analysis was conducted using the medical records of 768 patients with brain lesions between March 2013 and January 2017. The inclusion criteria for the study were adult patients ( $\geq 18$ years old) who had dysphagia and had been diagnosed with a brain lesion and referred for a VFSS and salivagram. We excluded patients who had more than a 1-week gap between the VFSS and radionuclide salivagram, as well as those who did not have a brain lesion, such as motor neuron disease, high cervical spinal cord injury and non-brain tumors. Patients with insufficient medical records were also excluded (Fig. 1).

\section{Videofluoroscopic swallowing study}

The study was performed with a fluoroscopy unit and recorded with computerized recording systems. All

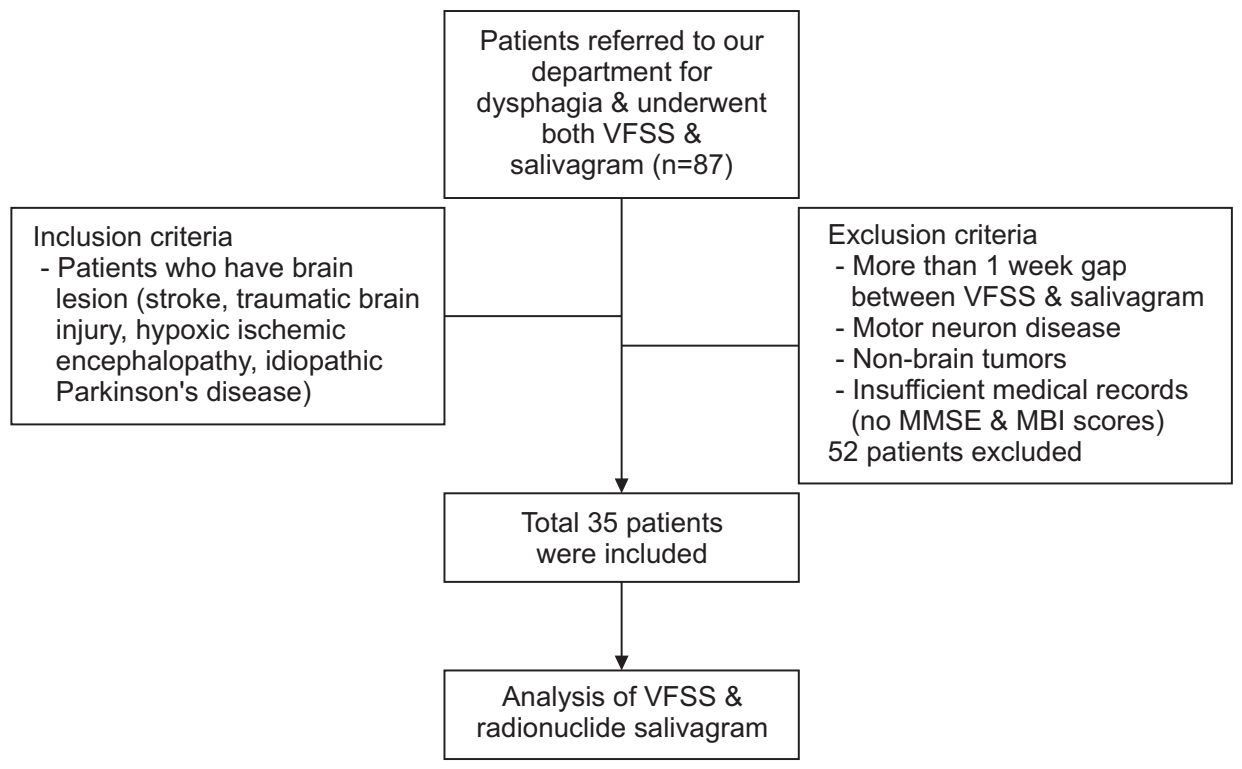

Fig. 1. Flow chart of inclusion and exclusion criteria of the study sample. VFSS, videofluoroscopic swallowing study; MMSE, MiniMental State Examination; MBI, Modified Barthel Index. 
studies were reviewed by two physiatrists. During the study patients were seated and sequentially swallowed the following materials that have a phased consistency mixed with liquid barium: water, yogurt, rice porridge, and boiled rice. The dynamic fluoroscopic images were obtained with lateral and anterior-posterior views and stored at 6 frames per second. The images were analyzed by one of the authors. The VFSS findings were scored according to the penetration-aspiration scale (PAS) [9] (Appendix 1) and classified as positive for aspiration if the PAS score was greater than 5 .

\section{Radionuclide salivagram}

Salivagrams were performed within a week from VFSS. All salivagrams were conducted in the Department of Nuclear Medicine at the Daegu Fatima Hospital. Patients were evaluated in a supine position under a gamma camera. A Tc-99m sulfur colloid solution $(0.5 \mathrm{~mL}$ of $0.3 \mathrm{mCi})$ was administered into the patient's mouth using a syringe. Sequential supine posterior images were obtained for 1 hour at 1, 5, 10, 20, 30, and 60 minutes after the solution was consumed. Images were taken by a gamma camera (102 Discovery NM630; GE Healthcare, Buckinghamshire, England). Visual interpretation of the images was performed by an experienced nuclear medicine physician. Aspiration was reported to be present when radiopharmaceutical activity was detected in the tracheobronchial fields (Fig. 2).

\section{Statistical analysis}

Group comparisons according to the findings of the radionuclide salivagram were performed using the Wilcoxon rank sum test or Mann-Whitney U-test and Pearson chi-square test. A multivariate logistic regression analysis with forward stepwise selection was performed to assess the association between the variables with statistically significant differences between the two groups and positive findings on the salivagram. Receiver operating characteristic (ROC) analyses were performed to assess the accuracy of the predictive factor for positive findings on the salivagram. Statistical analyses were performed using SPSS software ver. 22.0 (IBM, Armonk, NY, USA), and MedCalc program for Windows.

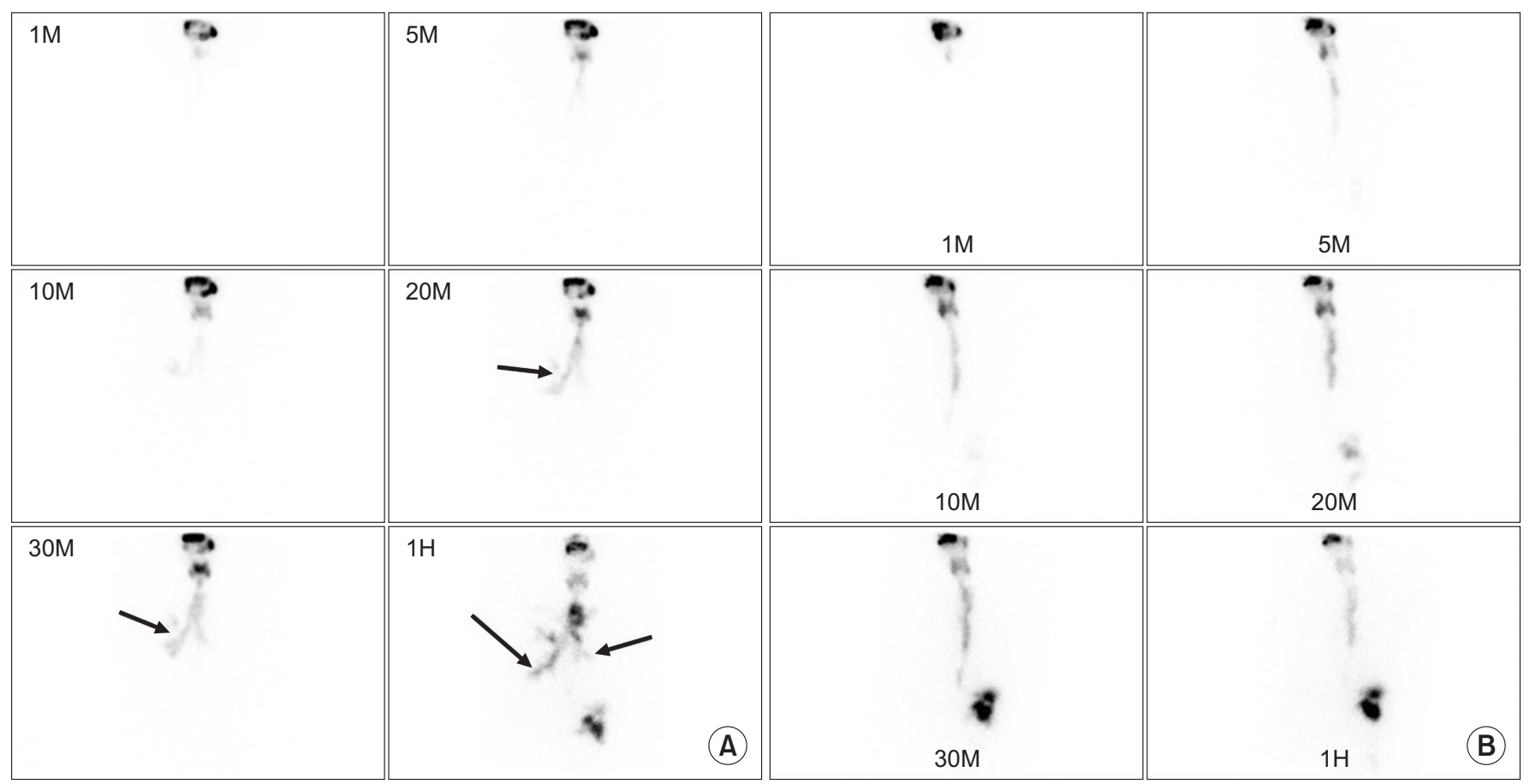

Fig. 2. Sequential images of the radionuclide salivagram throughout the 1 hour immediately following oral administration of the Tc-99m sulfur colloid solution. (A) The presence of a radiotracer in the trachea and bilateral bronchi is a positive indicator of salivary aspiration (arrows). (B) The uptake of the radiotracer noted only in the oropharynx, esophagus and stomach is a negative indicator of salivary aspiration. 
Table 1. Patient characteristics and the comparison of patients with and without aspiration on the radionuclide salivagram

\begin{tabular}{|c|c|c|c|}
\hline Characteristic & Aspiration (-) & Aspiration (+) & p-value \\
\hline Age (yr) & $69.75 \pm 12.505$ & $65.00 \pm 12.345$ & 0.294 \\
\hline Sex & & & 0.533 \\
\hline Male & $14(58.3)$ & $7(63.6)$ & \\
\hline Female & $10(41.7)$ & $4(36.4)$ & \\
\hline Duration of disease (day) & $157.4 \pm 633.1$ & $65.4 \pm 57.4$ & 0.057 \\
\hline \multicolumn{4}{|l|}{ Disease } \\
\hline Stroke & $24(96)$ & $7(63.6)$ & \\
\hline TBI & $0(0)$ & $3(27.3)$ & \\
\hline HIE & $0(0)$ & $1(9.1)$ & \\
\hline IPD & $1(4)$ & $0(0)$ & \\
\hline \multicolumn{4}{|l|}{ Involved brain region } \\
\hline Unilateral & 17 & 1 & $<0.001^{\text {b) }}$ \\
\hline Bilateral & 6 & 10 & \\
\hline \multicolumn{4}{|l|}{ VFSS } \\
\hline Penetraion or aspiration (+) & $24(68.57)$ & $11(31.43)$ & \\
\hline PAS (0-8) & $6.29 \pm 1.429$ & $6.91 \pm 1.514$ & 0.147 \\
\hline \multicolumn{4}{|l|}{ Feeding method } \\
\hline Oral feeding & 3 & 0 & $0.220^{\mathrm{b})}$ \\
\hline Nasogastric tubal feeding & 12 & 11 & \\
\hline MMSE & $21.27 \pm 5.709$ & $7.18 \pm 9.506$ & $0.001^{\mathrm{a})}$ \\
\hline GDS & $3.77 \pm 1.688$ & $5.55 \pm 1.508$ & $0.005^{\mathrm{a})}$ \\
\hline History of aspiration pneumonia & & & $0.046^{\mathrm{b})}$ \\
\hline$(-)$ & 19 & 5 & \\
\hline$(+)$ & 5 & 6 & \\
\hline \multicolumn{4}{|l|}{ MBI score } \\
\hline Total & $40.68 \pm 24.631$ & $13.11 \pm 18.584$ & $0.008^{\mathrm{a})}$ \\
\hline Personal hygiene & $3.00 \pm 1.247$ & $0.375 \pm 1.060$ & $<0.001^{\text {a) }}$ \\
\hline Bathing & $2.68 \pm 1.293$ & $0.25 \pm 0.463$ & $<0.001^{\text {a) }}$ \\
\hline Feeding & $5.47 \pm 2.836$ & $0.88 \pm 1.808$ & $0.001^{\mathrm{a})}$ \\
\hline Toilet & $4.16 \pm 2.853$ & $0.50 \pm 0.926$ & $<0.001^{\text {a) }}$ \\
\hline Stair climbing & $1.37 \pm 2.813$ & 0 & 0.169 \\
\hline Dressing & $4.36 \pm 2.872$ & $0.88 \pm 1.808$ & $0.001^{\mathrm{a})}$ \\
\hline Defecation & $6.84 \pm 3.594$ & $2.13 \pm 3.643$ & $0.007^{\mathrm{a})}$ \\
\hline Voiding & $5.11 \pm 4.332$ & $0.63 \pm 1.768$ & $0.006^{\mathrm{a})}$ \\
\hline Ambulation & $3.26 \pm 4.395$ & $0.38 \pm 1.061$ & 0.050 \\
\hline Wheelchair & $0.32 \pm 0.478$ & $0.63 \pm 1.061$ & 0.629 \\
\hline Chair/bed transfer & $6.58 \pm 3.761$ & $1.50 \pm 1.604$ & $0.001^{\mathrm{a})}$ \\
\hline
\end{tabular}

Values are presented as mean \pm standard deviation or number (\%).

TBI, traumatic brain injury; HIE, hypoxic ischemic encephalopathy; IPD, idiopathic Parkinson's disease; VFSS, videofluoroscopic swallowing study; PAS, penetration-aspiration scale; MMSE, Mini-Mental State Examination; GDS, Global Deterioration Scale; MBI, Modified Barthel Index.

${ }^{a)} \mathrm{p}<0.05$ by Wilcoxon rank sum test or Mann-Whitney U-test.

${ }^{b)} \mathrm{p}<0.05$ by Pearson chi-square test. 


\section{RESULTS}

\section{Patient characteristics}

A total of 87 patients with brain lesions were referred for swallowing evaluation. Thirty-five patients who satisfied our criteria were included in this study. Their demographic data are presented in Table 1. Subjects were divided into two groups according to the presence of aspiration on the salivagram. Eleven out of 35 patients had documented salivary aspiration on the radionuclide salivagram. Only one patient with positive aspiration on the salivagram had a negative aspiration finding on the VFSS.

\section{Comparison between patients with and without aspiration on the salivagram}

Age, sex, duration of disease, PAS, Mini-Mental State Examination (MMSE), Global Deterioration Scale (GDS), total and each item of score of Modified Barthel Index (MBI) were compared between the two groups, using the Wilcoxon rank sum test or Mann-Whitney U-test. Statistically significant differences were observed in the following variables: MMSE, GDS, total and many itemwise MBI score $(\mathrm{p}<0.05$, Table 1$)$. The differences in sex, feeding method and incidence of aspiration pneumonia between the two groups were analyzed by a Pearson chi-squared test. There were no statistically significant differences in sex $(\mathrm{p}=0.533)$ and feeding method $(\mathrm{p}=0.220)$ between the two groups. However, the incidence of aspiration pneumonia was significantly high in the group that had documented aspiration on the salivagram $(p=0.046)$. The distribution ratio of patients that had hemiplegia or quadriplegia in two groups was also assessed. There was a statistically significant difference in the distribution of quadriplegia and hemiplegia between the two groups. There were more quadriplegic patients in the group that had positive findings on the salivagram.

A multivariate logistic regression analysis with a forward stepwise method demonstrated that only the MMSE score was significantly associated with positive findings on the salivagram (odds ratio $=0.760 ; 95 \%$ confidence in- terval [CI], 0.625-0.923; $\mathrm{p}=0.006$ ) (Table 2).

MMSE score and aspiration on the radionuclide salivagram

In the ROC curve analysis, the area under the ROC curve (AUC) for developing aspiration on the salivagram was 0.855 (95\% CI, 0.689-0.953; p<0.0001) (Fig. 3). The optimal cut-off values obtained from the maximal Youden index was a MMSE score of 7 (sensitivity 72.73\%, specificity $100 \%$ ) for performing radionuclide salivagram.

\section{DISCUSSION}

Aspiration pneumonia is a major cause of death in patients with brain lesions. Aspiration may result from swallowing dysfunction, gastroesophageal reflux, or inappropriate passage to the airway of oral secretion $[4,10]$.

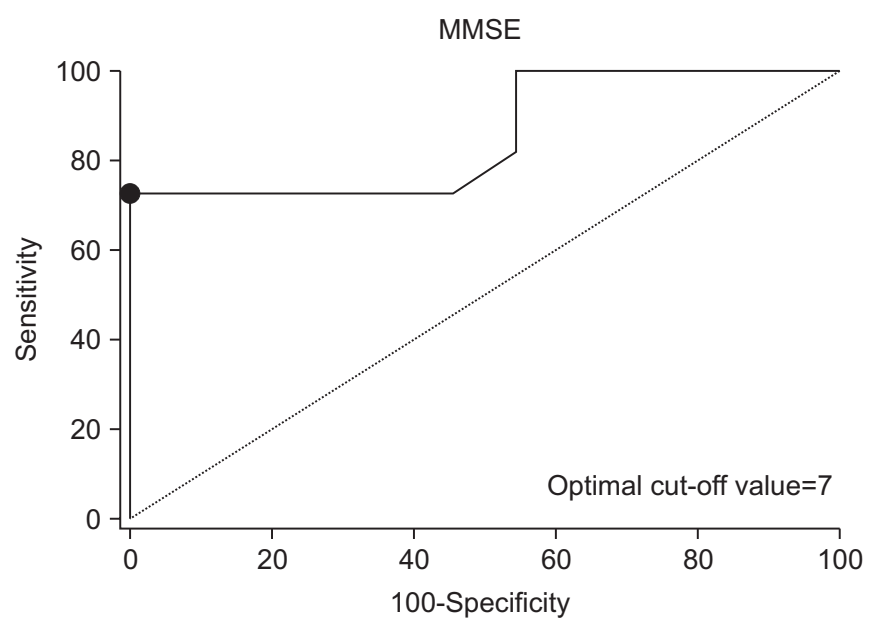

Fig. 3. ROC curve of MMSE score for developing aspiration on the radionuclide salivagram in patients with brain lesions. The optimal cut-off value (dots on the curves) for the MMSE score, which was obtained from the maximal Youden index, was a value of 7 for aspiration on the radionuclide salivagram (AUC $=0.855$; 95\% CI, 0.689-0.953; p $<0.0001$; sensitivity $72.73 \%$, specificity $100 \%$ ). ROC, receiver operating characteristic; MMSE, Mini-Mental State Examination; AUC, area under the ROC curve.

Table 2. Multivariate logistic regression analysis with forward stepwise method of clinical characteristics associated with aspiration on radionuclide salivagram

\begin{tabular}{ccccc}
\hline Parameter & Beta coefficient & Standard error & OR (95\% CI) & p-value \\
\hline MMSE score & -0.275 & 0.100 & $0.760(0.625-0.923)$ & 0.006 \\
\hline
\end{tabular}

OR, odds ratio; CI, confidence interval; MMSE, Mini-Mental State Examination. 
In evaluating aspiration during swallowing, a VFSS is one of the important and appropriate assessment tools, especially for evaluating aspiration of food and liquids. However, saliva swallowing is different because their minimal volume is difficult to test in VFSS and saliva swallowing occurs spontaneously and reflexively without effort. Although the amount of saliva aspiration is small, there is a pathogen colonization of the oral cavity and pharyngeal mucosa [11] that can lead to aspiration pneumonia. Furthermore, patients with brain lesions are more susceptible to saliva aspiration because of their conditions, reduced mental status, supine position, poor oral hygiene, decreased salivary clearance and oromotor dysfunctions. Accordingly, a radionuclide salivagram may impose its role in evaluating potential saliva aspiration in patients with brain lesions.

In a previous study, a radionuclide salivagram was more sensitive in detecting aspiration than VFSS [12]. However, in our study, the rate of aspiration was higher in VFSS. The difference between the two studies may be due to the difference in the subjects and examination methods in the radionuclide salivagram. The previous study included children with severe non-ambulant cerebral palsy and used a larger amount of solution than our study (20 mL of Tc-99m sulfur colloid solution) [12]. Moreover, the radiopharmaceutical solution was instilled into the children's mouth continuously for 1 hour. Whereas, our study included adult patients with brain lesions, and only $0.5 \mathrm{~mL}$ of the solution was injected into the patient's mouth at once. Afterwards, we waited for swallowing to occur. Other previous studies involving salivagrams have reported positive results for aspiration more frequently in the groups who were given a high volume of radionuclide bolus than the groups who were given a low volume $[7,13]$. In this regard, we attempted to confirm the aspiration of saliva itself in the most natural way without affecting saliva production and inducing an artificial swallowing response by administering a large volume of bolus.

Previously, many studies on radionuclide salivagrams have focused on the child population $[7,8,12,13]$. There are not many studies that involve the adult population $[14,15]$, and only a few studies that report the utility of salivagrams in patients with brain lesions [15-17]. Moreover, there has been no studies that identify the correlation between the clinical features of the patients and salivary aspiration.
Of the total 35 patients included in our study, there were 24 patients $(68.57 \%$ ) who had negative findings on the salivagram but confirmed aspiration and penetration in the VFSS (patients with unmatched results in both test), and there were 11 patients (31.43\%) who had positive findings on the salivagram and VFSS simultaneously (Table 1). In a previous study that compared the VFSS and salivagram, there were 5 patients $(29.4 \%)$ who had negative findings on the salivagram and positive findings on the VFSS, and there were 9 patients (27.3\%) who confirmed aspiration on the salivagram and VFSS simultaneously, which is in contrast to the results of this study [18]. However, the previous study included patients with aspiration pneumonia in a heterogeneous disease group and not necessarily patients with brain lesions [18]. Therefore, the value of this study includes it being the first to define the correlation between salivary aspiration and the clinical characteristics of patients with brain lesions. In this study, there was significant correlation between cognition and salivary aspiration, but no significant correlation between the total MBI score, which is an indicator of a patient's functional disability, and salivary aspiration. Considering the results of this study that suggests that salivary aspiration is more affected by the cognition of the patient with brain lesions rather than the functional status, it may be advantageous to perform a radionuclide salivagram routinely in patients with low MMSE scores to predict aspiration pneumonia.

In patients with salivary aspiration confirmed by a salivagram, the possibility of continuous salivary aspiration should be considered even when an oral diet is limited. Additionally, to prevent saliva aspiration in any bedridden patients, including patients with brain lesions, the semi-reclined postural measure with chest physical therapy or occupational therapy activities with oromotor facilitation may be helpful, if possible and permitted. In addition to reducing the risk of aspiration by modification of the patient's diet and rehabilitation treatment for dysphagia, treatments to reduce salivary production also needs to be considered. Methods for controlling salivary production include use of anticholinergics, botulinum neurotoxin A (BoNT-A) injections into the salivary glands, radiation therapy, and surgery $[19,20]$.

There are several limitations of this study. First, because the number of patients studied was not sufficiently large, we could not classify and assess patients according 
to type or location of brain lesion, especially the brain stem which highly influences the swallowing function. In the positive finding on the salivagram group, 10 out of the 11 participants were quadriplegic. There was a significant difference in the distribution of quadriplegia and hemiplegia between patients with and without aspiration on the salivagram. In the multivariate model, however, the variables other than cognitive impairment assessed by the MMSE, such as the total MBI score, were not significantly correlated with the findings of the salivagram. Future studies with larger patients group are needed to certify the results of this study. Finally, we excluded patients with insufficient medical records, which may have introduced bias in the analysis. In addition, incomplete medical records for patients that were included in this study led to insufficient covariates in the multivariate logistic regression analysis. The insufficient covariates were largely due to the retrospective nature of this study.

In patients with brain lesions who complained of dysphagia, the MMSE score was correlated with salivary aspiration. For patients with a score of 7 or less on the MMSE, it may be helpful to perform a radionuclide salivagram for the early detection of patients at high risk for aspiration pneumonia induced from salivary aspiration. For patients who have confirmed salivary aspiration, the treatment described in this study will help reduce mortality and morbidity associated with aspiration pneumonia.

\section{CONFLICT OF INTEREST}

No potential conflict of interest relevant to this article was reported.

\section{REFERENCES}

1. Vermeij FH, Scholte op Reimer WJ, de Man P, van Oostenbrugge RJ, Franke CL, de Jong G, et al. Strokeassociated infection is an independent risk factor for poor outcome after acute ischemic stroke: data from the Netherlands Stroke Survey. Cerebrovasc Dis 2009; 27:465-71.

2. Roger VL, Go AS, Lloyd-Jones DM, Benjamin EJ, Berry JD, Borden WB, et al. Heart disease and stroke statistics: 2012 update: a report from the American Heart Association. Circulation 2012;125:e2-220.
3. Marik PE. Pulmonary aspiration syndromes. Curr Opin Pulm Med 2011;17:148-54.

4. Boesch RP, Daines C, Willging JP, Kaul A, Cohen AP, Wood RE, et al. Advances in the diagnosis and management of chronic pulmonary aspiration in children. Eur Respir J 2006;28:847-61.

5. Aviv JE, Sacco RL, Mohr JP, Thompson JL, Levin B, Sunshine S, et al. Laryngopharyngeal sensory testing with modified barium swallow as predictors of aspiration pneumonia after stroke. Laryngoscope 1997; 107:1254-60.

6. Croghan JE, Burke EM, Caplan S, Denman S. Pilot study of 12-month outcomes of nursing home patients with aspiration on videofluoroscopy. Dysphagia 1994;9:141-6.

7. Heyman S, Respondek M. Detection of pulmonary aspiration in children by radionuclide "salivagram". J Nucl Med 1989;30:697-9.

8. Heyman $\mathrm{S}$. The radionuclide salivagram for detecting the pulmonary aspiration of saliva in an infant. Pediatr Radiol 1989;19:208-9.

9. Rosenbek JC, Robbins JA, Roecker EB, Coyle JL, Wood JL. A penetration-aspiration scale. Dysphagia 1996;11:93-8.

10. Lee ZI, Cho DH, Choi WD, Park DH, Byun SD. Effect of botulinum toxin type a on morphology of salivary glands in patients with cerebral palsy. Ann Rehabil Med 2011;35:636-40.

11. Li X, Kolltveit KM, Tronstad L, Olsen I. Systemic diseases caused by oral infection. Clin Microbiol Rev 2000;13:547-58.

12. Baikie G, South MJ, Reddihough DS, Cook DJ, Cameron DJ, Olinsky A, et al. Agreement of aspiration tests using barium videofluoroscopy, salivagram, and milk scan in children with cerebral palsy. Dev Med Child Neurol 2005;47:86-93.

13. Heyman S. Volume-dependent pulmonary aspiration of a swallowed radionuclide bolus. J Nucl Med 1997; 38:103-4.

14. Silver KH, Van Nostrand D. The use of scintigraphy in the management of patients with pulmonary aspiration. Dysphagia 1994;9:107-15.

15. Nakagawa T, Sekizawa K, Arai H, Kikuchi R, Manabe K, Sasaki H. High incidence of pneumonia in elderly patients with basal ganglia infarction. Arch Intern Med 1997;157:321-4. 
16. Kang IS, Kwon JG, Lee SU, Lee ZI, Park GY, Park HW. Detection of saliva aspiration using salivagram in bedridden patients with brain lesion. J Korean Acad Rehabil Med 2010;34:503-7.

17. Kang Y, Chun MH, Lee SJ. Evaluation of salivary aspiration in brain-injured patients with tracheostomy. Ann Rehabil Med 2013;37:96-102.

18. Jang DH, Choi KH, Kim DH, Lim CM, Kim JS. Comparison between the radionuclide salivagram and videofluoroscopic swallowing study methods for evaluating patients with aspiration pneumonia. Ann Nucl
Med 2013;27:247-52.

19. Meningaud JP, Pitak-Arnnop P, Chikhani L, Bertrand JC. Drooling of saliva: a review of the etiology and management options. Oral Surg Oral Med Oral Pathol Oral Radiol Endod 2006;101:48-57.

20. Lee ZI, Yu KJ, Lee DH, Hong SK, Woo SB, Kim JM, et al. The effect of nebulized glycopyrrolate on posterior drooling in patients with brain injury: two cases of different brain lesions. Am J Phys Med Rehabil 2017;96: e155-8. 
Appendix 1. The penetration-aspiration scale (PAS)

\begin{tabular}{lcll}
\hline \multicolumn{1}{c}{ Category } & Score & Description \\
\hline Negative & 1 & Material does not enter the airway \\
Penetration & 2 & Material enters the airway, remains above the vocal folds, and is ejected from the airway \\
& 3 & Material enters the airway, remains above the vocal folds, and is not ejected from the airway \\
& 4 & Material enters the airway, contacts the vocal folds, and is ejected from the airway \\
Aspiration & 5 & $\begin{array}{l}\text { Material enters the airway, contacts the vocal folds, and is not ejected from the airway } \\
\text { Material enters the airway, passes below the vocal folds, and is ejected into the larynx or out } \\
\text { of the airway }\end{array}$ \\
& 7 & $\begin{array}{c}\text { Material enters the airway, passes below the vocal folds, and is not ejected from the trachea } \\
\text { despite effort } \\
\text { Material enters the airway, passes below the vocal folds, and no effort is made to eject }\end{array}$ \\
\hline
\end{tabular}

